\title{
LA-UR-18-24486
}

Approved for public release; distribution is unlimited.

Title: A systematic error affecting NER calculations

Author(s): $\quad$ Mulford, Roberta Nancy

Intended for: $\quad$ Report

Issued: 
Disclaimer:

Los Alamos National Laboratory, an affirmative action/equal opportunity employer, is operated by the Los Alamos National Security, LLC for the National Nuclear Security Administration of the U.S. Department of Energy under contract DE-AC52-06NA25396. By approving this article, the publisher recognizes that the U.S. Government retains nonexclusive, royalty-free license to publish or reproduce the published form of this contribution, or to allow others to do so, for U.S. Government purposes. Los Alamos National Laboratory requests that the publisher identify this article as work performed under the auspices of the U.S. Department of Energy. Los Alamos National Laboratory strongly supports academic freedom and a researcher's right to publish; as an institution, however, the Laboratory does not endorse the viewpoint of a publication or guarantee its technical correctness. 


\section{A systematic error affecting NER calculations}

Roberta N. Mulford

17 May 2018

Factors that may affect the precision of Neutron Emission Rate (NER) measurements made in the Canberra 4 neutron counter have been examined and quantified. [1] Measurements of sample NER are corrected for the efficiency of the counter. The efficiency is calculated by measuring a source of known intensity, and taking the ratio of the measured neutrons per second $(n / s)$ to the known output of the source.

A standard ${ }^{252} \mathrm{Cf}$ sample is used to determine the efficiency of a neutron counter. The efficiency of the counter is less than 1 for several reasons. The gate time of the detectors is finite, the cross section of the detectors precludes capture of all emitted neutrons, not all neutrons are thermalized in the distance between the source and the detectors, and the cylindrical array of detectors occupies only a fraction of the sphere into which the source emits neutrons. The efficiency of the neutron counter is determined before each measurement by measuring a ${ }^{252} \mathrm{Cf}$ sample of known initial neutron output and age. The current neutron output from the calibrated source on the day of measurement is calculated from the half-life of ${ }^{252} \mathrm{Cf}$, and the source output on the day of calibration. The ratio of the measured neutron output to the calculated neutron output provides a value for instrument efficiency. This value of efficiency should be a characteristic of the neutron counter and should be constant from day to day. Efficiency is measured on each day when measurements are performed, to identify perturbations such as nearby sources in the environment of the counter before each measurement. Calculated efficiency of the NER appears to depend to some extent on the absolute output of the calibration source, as shown in Figure 1.

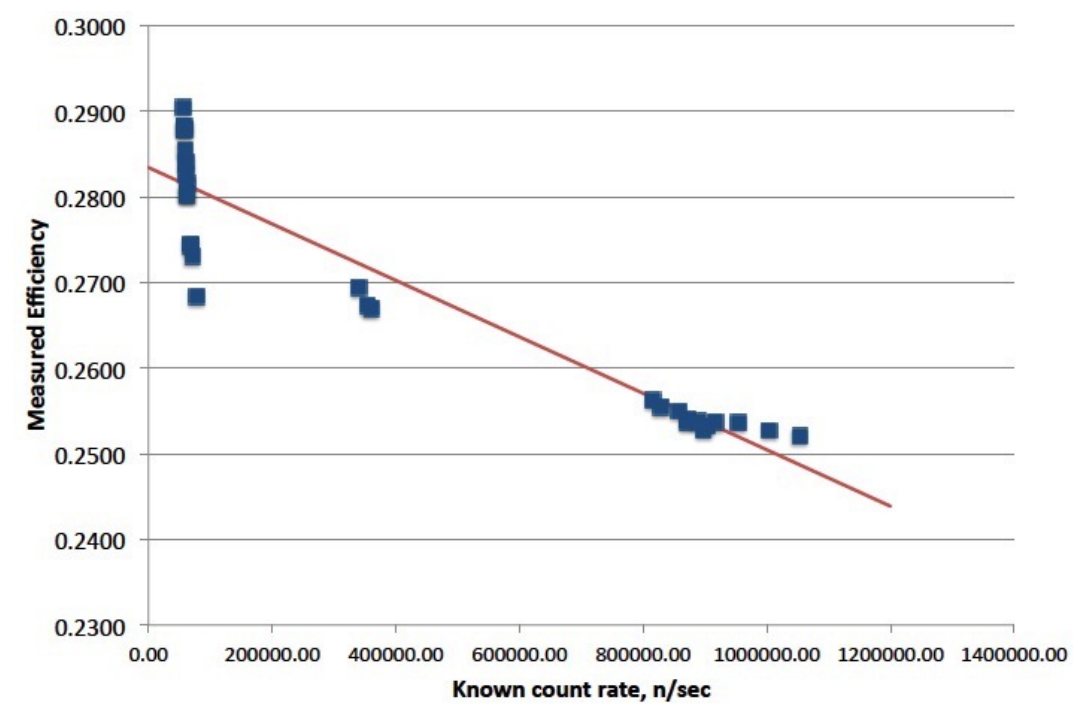

Figure 1. Calculated efficiency as a function of neutron source intensity 
This dependence of efficiency on source intensity results in a systematic error in the measurement, a dependence on the neutron output of the sample being measured. For fuel samples, measured values are corrected using the efficiency determined from measurements on a standard source which has count rates near 60,000 n/s.

For the source Z214 with output around 60,000 n/sec, the dependence of efficiency on neutron output appears to be stronger than the dependence determined over the range from $60,000 \mathrm{n} / \mathrm{s}$ to $1,000,000 \mathrm{n} / \mathrm{sec}$. The dependence of efficiency on source output for source Z214 is shown in Figure 2.

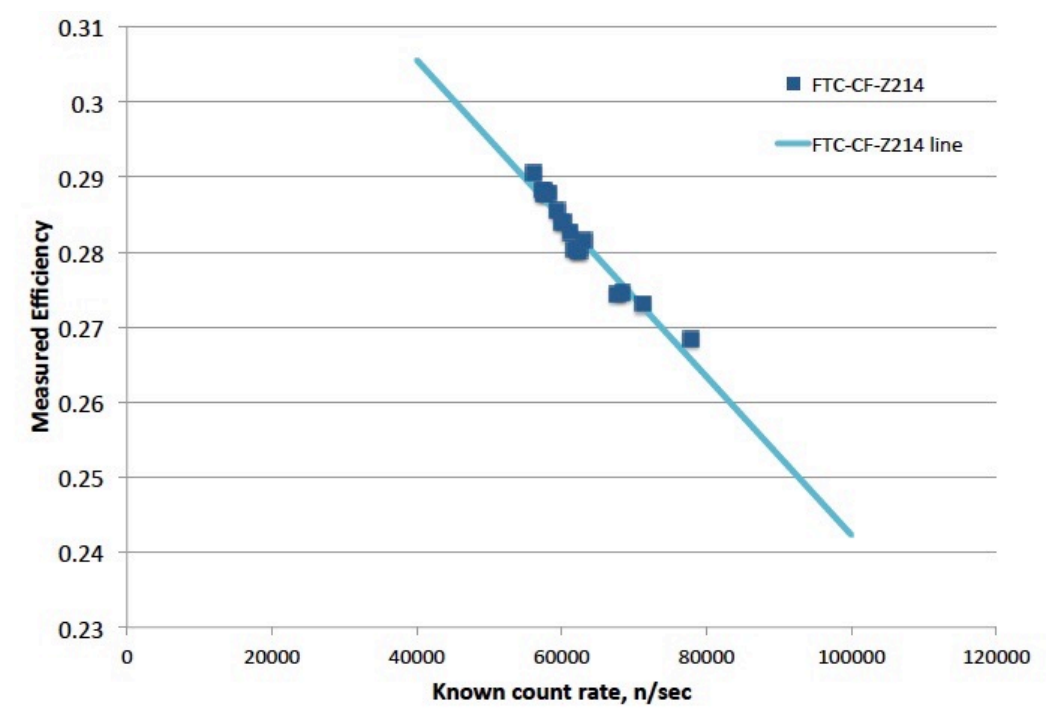

Figure 2. Dependence of efficiency on neutron output of source Z214 with intensities on the order of $60,000 \mathrm{n} / \mathrm{s}$. Slope is $-1.07 \mathrm{E}-6$ /count.

A similar dependence of calculated efficiency on sample output can be determined for other samples, as shown in Figures 3 and 4. Dependencies differ for different samples, as shown in Figures 2-4. 


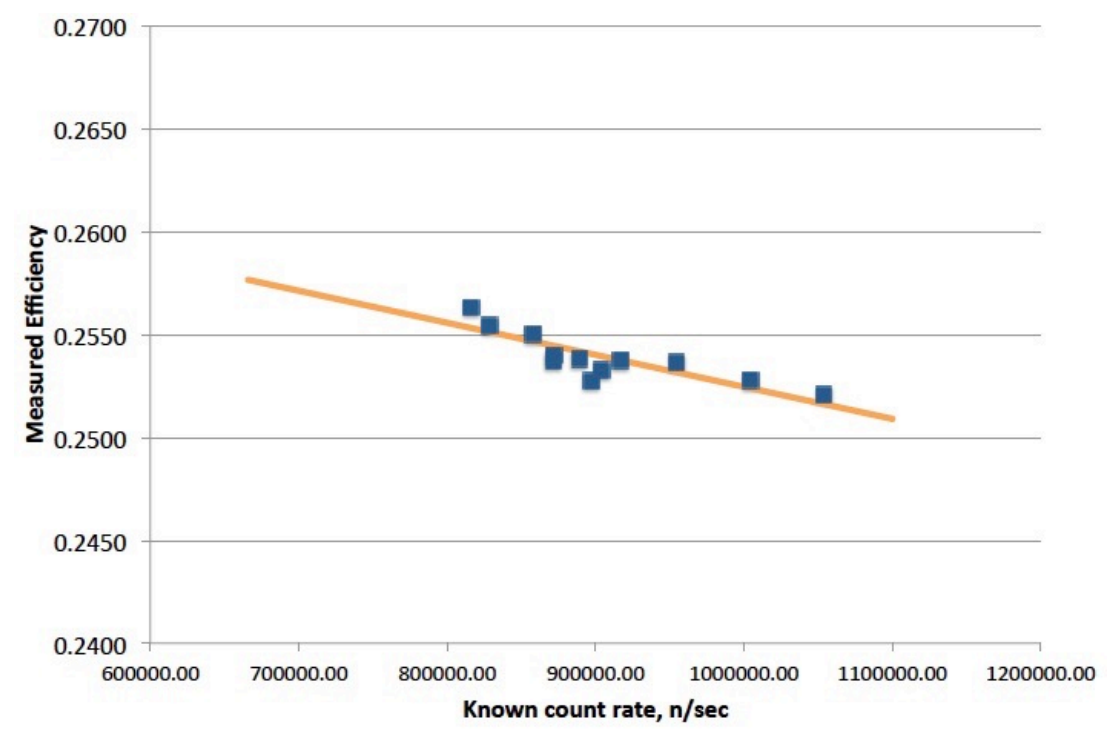

Figure 3. Dependence of efficiency on neutron output of source Z1969 with intensities on the order of $900,000 \mathrm{n} / \mathrm{s}$. Slope is $-1.55 \mathrm{E}-8 /$ count.

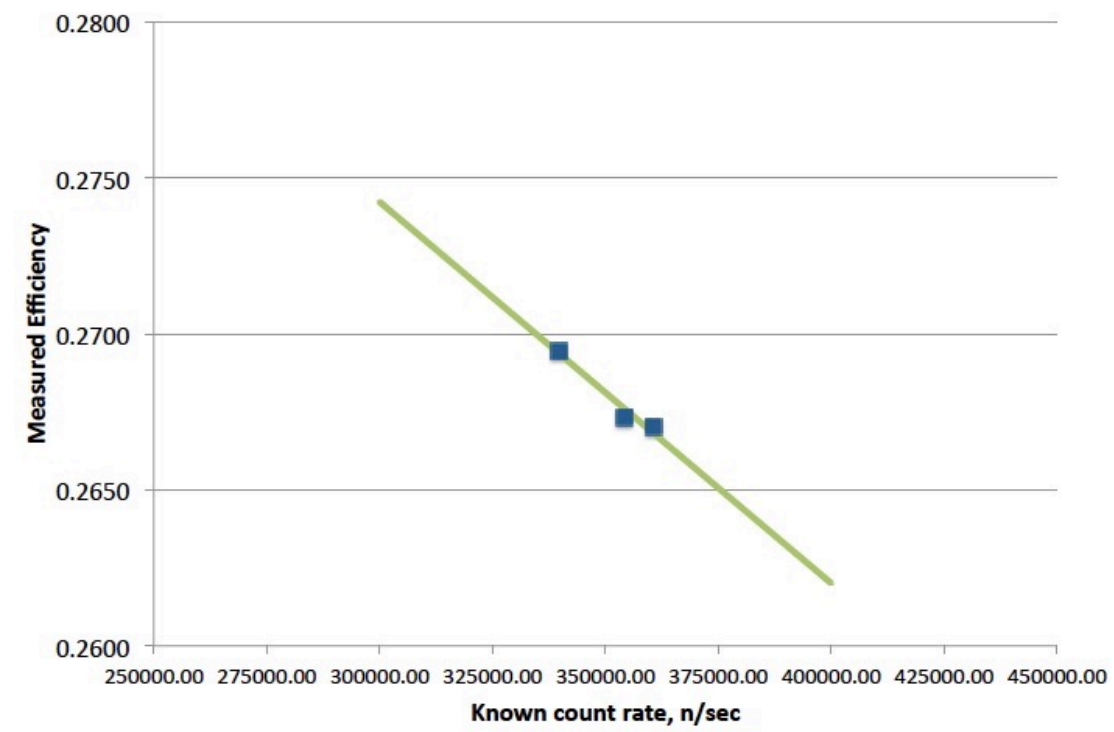

Figure 4. Dependence of efficiency on neutron output of source Z1962 with intensities on the order of 350,000 n/s. Slope is $-1.22 \mathrm{E}-7 /$ count.

\section{Determining the ${ }^{250} \mathrm{Cf}$ content of a source}

Any ${ }^{252} \mathrm{Cf}$ sample contains some proportion of ${ }^{250} \mathrm{Cf}$, which has a long half-life. Thus, when we calculate a current output assuming that the entire sample is ${ }^{252} \mathrm{Cf}$, the value calculated is too low, because of the persistent fraction of ${ }^{250} \mathrm{Cf}$. The neutron output of the ${ }^{250} \mathrm{Cf}$ serves as a slowly varying baseline adding counts to the neutron output of the sample, with a proportion that increases with time as the ${ }^{252} \mathrm{Cf}$ decays. 
The ${ }^{250} \mathrm{Cf}$ content of the sample is evident when the dependence of the efficiency on neutron output of the standard sample Z214 is plotted, as in Figure 2.

In order to correct the calculated source decay for emission from both the ${ }^{252} \mathrm{Cf}$ and the ${ }^{250} \mathrm{Cf}$ isotope, the ${ }^{250} \mathrm{Cf}$ content of the source must first be determined.

The output of a mixed sample can be calculated by taking into account the half-lives of both isotopes:

${ }^{252} \mathrm{Cf} \quad 966.1$ days

${ }^{250} \mathrm{Cf} \quad 4777.47$ days

At any time t, the count rate $\mathrm{N}$ is expressed in term of the initial count rate $\mathrm{N}_{0}$ as

$\mathrm{N}=x \mathrm{~N}_{0} \mathrm{e}^{-\lambda 252 \mathrm{t}}+(x-1) \mathrm{N}_{0} \mathrm{e}^{-\lambda 250 \mathrm{t}} \quad$ Equation 1.

In Equation 1, $N$ is the neutron output of the source at time $t$ after calibration, $N_{0}$ is the output of the source at calibration, $x$ is the fraction of the sample that is composed of ${ }^{252} \mathrm{Cf}$ and $(x-1)$ is the fraction of ${ }^{250} \mathrm{Cf}$. The parameter $\lambda_{252}$ is the decay contant, defined

$\lambda 252=\ln 2 / \tau_{1 / 2}$ Equation 2.

where $\tau_{1 / 2}$ is the half-life of the ${ }^{252} \mathrm{Cf}$ isotope, and similarly $\lambda_{250}$ for the ${ }^{250} \mathrm{Cf}$ isotope.

The formula in Equation 1 can be reduced in various ways. [2] Values reported here are determined by varying the ${ }^{250} \mathrm{Cf}$ content $X$ in Equation 1 and minimizing the average deviation between calculated and observed values of neutron output. Fractions determined are reported in Table 1.

Table 1. Properties of Measured Sources

\begin{tabular}{|l|c|c|c|}
\hline Source & $\mathrm{Z} 214$ & $\mathrm{Z} 1969$ & $\mathrm{Z} 1962$ \\
\hline Fraction ${ }^{252} \mathrm{Cf}$ & 0.722 & 0.9519 & 0.9095 \\
\hline Fraction ${ }^{250} \mathrm{Cf}$ & 0.278 & 0.0481 & 0.0905 \\
\hline $\begin{array}{l}\text { Calibrated neutron } \\
\text { output, } \mathrm{n} / \mathrm{s}\end{array}$ & 83825 & 1218234 & $1.2 \mathrm{E}+06$ \\
\hline Calibration date & $9 / 30 / 16$ & $9 / 30 / 16$ & $12 / 12 / 11$ \\
\hline
\end{tabular}


Uncorrected and corrected efficiencies are shown in Figure 6. The machine efficiency as determined using each source is shown in Figure 6.

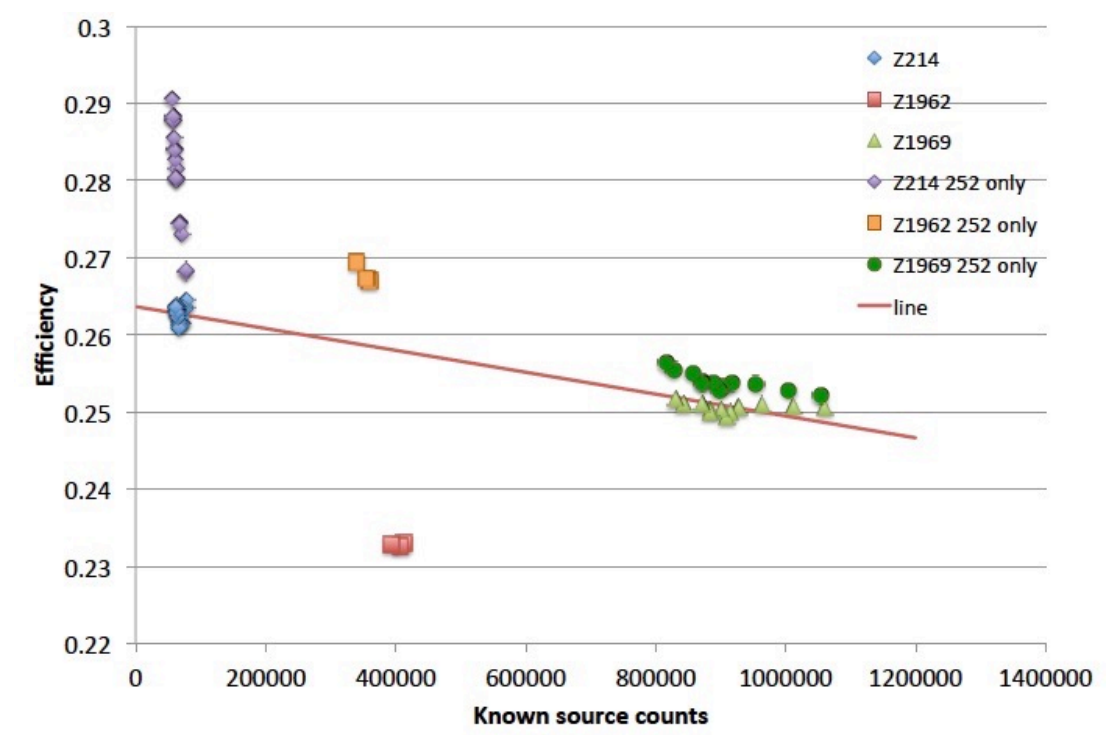

Figure 6. Machine efficiency determined using both ${ }^{252} \mathrm{Cf}$ and ${ }^{250} \mathrm{Cf}$ output from three sources. Slope is $-1.4 \mathrm{E}-8 /$ count.

A slight dependency of efficiency on neutron output can be seen by comparing the corrected values obtained using source Z1969 with the corrected values obtained using the source Z214. This may indicate a real dependency of the efficiency of detection as a function of neutron output, which may result from the finite gate time of the detector, from the finite thickness of the moderator, or from the detector geometry. A real dependency would lead to a slight error in determination of source composition.

The efficiencies obtained using source Z1962 fall well below efficiencies obtained using the other two sources. The composition of source Z1962 was determined from only three measured values, which may be insufficient to accurately define the composition.

Variation in the corrected efficiencies determined using source Z214 is shown in Figure 7. 


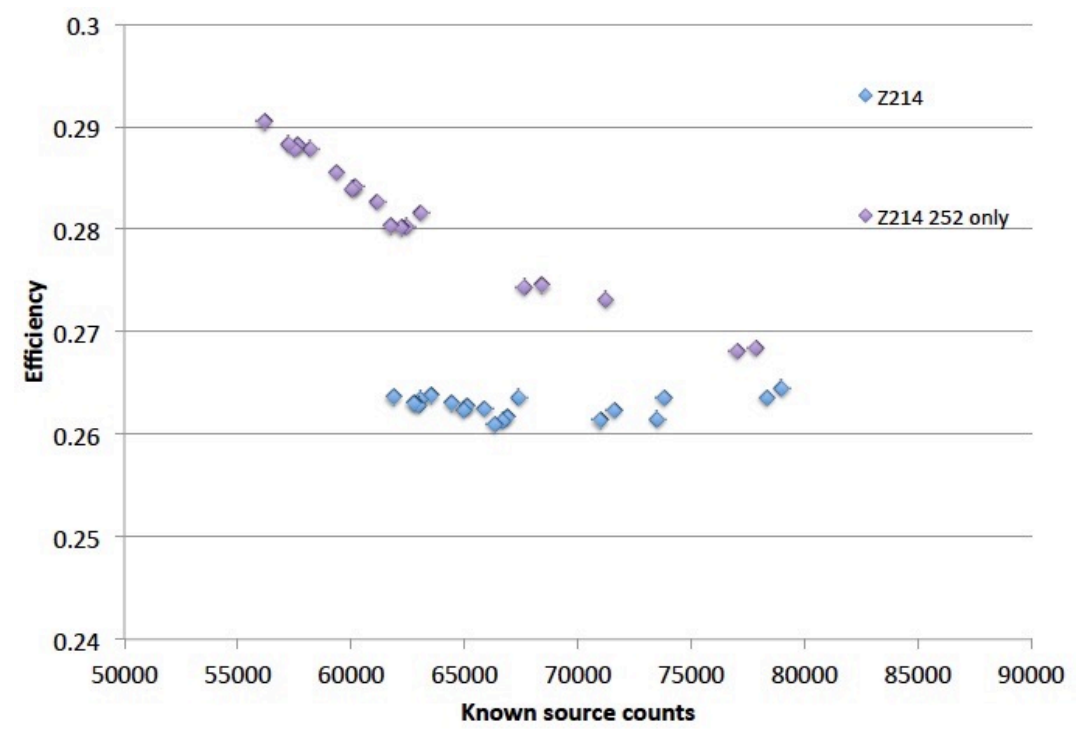

Figure 7. Machine efficiency determined using only both ${ }^{252} \mathrm{Cf}$ (purple) and both ${ }^{252} \mathrm{Cf}$ and ${ }^{250} \mathrm{Cf}$ output (blue) from source $\mathrm{Z} 214$.

Attention to the possibility that age-correction of the neutron output of a calibrated source may yield a slightly perturbed value for total neutron output is necessary to ensure the most accurate measurements of NER, particularly for samples with low neutron outputs. A nominally ${ }^{252} \mathrm{Cf}$ source may need to be corrected for the ${ }^{250} \mathrm{Cf}$ content of the calibration source. This correction can be made once several values of efficiency have been determined at differing source ages, and should be made for every calibration source, once sufficient data has been accrued.

The economics of californium source calibration and resale suggest that sources with lower absolute neutron output have probably been recalibrated and resold a number of times, and may be older and correspondingly lower in ${ }^{252} \mathrm{Cf}$ than sources with higher neutron outputs. The ${ }^{250} \mathrm{Cf}$ may comprise a non-trivial fraction of the nuclear material in such sources. The ${ }^{250} \mathrm{Cf}$ content as a function of the initial output of these three sources is shown in Figure 8. 


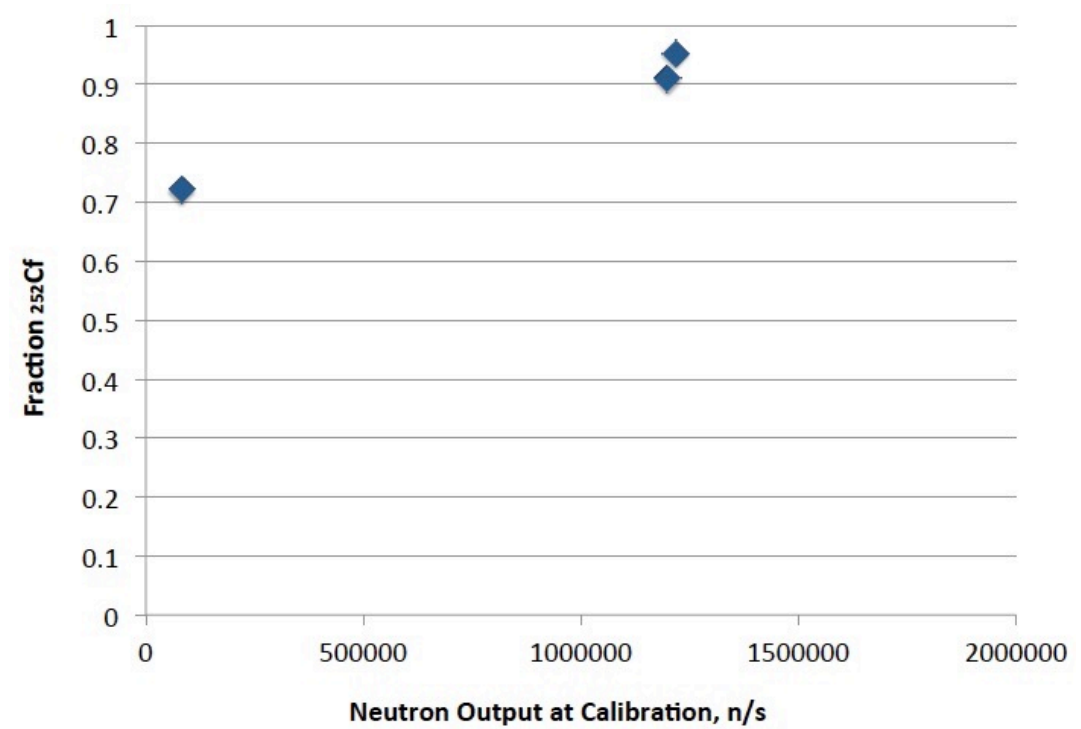

Figure $8 .{ }^{250} \mathrm{Cf}$ content of sources as a function of initial (calibrated) source output

The application of an uncorrected efficiency to values determined for samples can lead to a large error in the measured sample output, particularly for sample with small neutron outputs.

Over the narrow range between $77854 \mathrm{n} / \mathrm{s}$ and $56172 \mathrm{n} / \mathrm{s}$ the uncorrected efficiency varies from 0.2684 and 0.2905 . Uncorrected efficiency over this range varies as

Efficiency $=-1.067 \mathrm{E}-06 *($ count rate $)+0.3478$

If the efficiency determined from source Z214 is applied to a sample with an output of $2000 \mathrm{n} / \mathrm{s}$, the adjusted efficiency would be 0.3457 , rather than the value of about 0.2883 derived from source Z214. The difference is 0.0574 , or $20 \%$. This error dominates the $7.9 \%$ uncertainty arising primarily from the calibration of the standard source. The calculated NER of fuel samples is $20 \%$ too low if the dependence on efficiency observed over the narrow range around 60,000 n/s persists to lower neutron outputs.

For this reason, the efficiency calculated from source Z214 should be determined using the half-lives of both isotopes ${ }^{252} \mathrm{Cf}$ and ${ }^{250} \mathrm{Cf}$.

\section{References}

1. J. Wendelberger, "Propagation of Variance (POV) Uncertainty Calculations for the Neutron Emission Rate (NER) Measurement Process," Los Alamos National Laboratory LA-CP-18-20263 (April 2018.)

2. N.J. Roberts and L.N. Jones, Radiation Protection Dosimetry, v. 126 (1-4) pp. 8388 (August 2007.) 